\title{
Interaction between motor vehicles and bicycles at two-lane roundabouts: a driving volatility based analysis
} Ph.D. Mechanical Engineering - Transportation

\author{
Behnam Bahmankhah, Paulo Fernandes, João Teixeira, Margarida C. Coelho \\ Department of Mechanical Engineering \\ Centre for Mechanical Technology and Automation (TEMA) \\ Aveiro, Portugal \\ behnam.bahmankhah@ua.pt,paulo.fernandes@ua.pt,jpteixeira@ua.pt, margarida.coelho@ua.pt
}

\begin{abstract}
Although safety benefits of roundabouts for motor vehicles are well-documented [1], these are not always extensive to bicycles [2]. One of the main reasons may be the unexpected driving volatility of motor vehicle (MV)-bicycle interaction at roundabouts. Drivers' instantaneous decisions regarding speed and acceleration/deceleration, as well as the time rate of acceleration change (jerk) can result in a volatility driving behavior with significant impact on cyclist safety $[3,4]$. The main objective of this paper is the assessment of driving volatility in MV-bicycle interactions at two-lane roundabouts and its impacts on safety, pollutant emissions and traffic performance. Traffic flow and bicycle GPS data were collected from two two-lane roundabouts in the city of Aveiro, Portugal. Based on field measurements, a description of the different operational and behavior variables, such as entry and exit traffic flows, bicycle volumes, acceleration-deceleration rates and unexpected maneuvers experienced by drivers and cyclists was developed. Then, traffic (VISSIM), emissions (VSP) and safety (SSAM) models were used to evaluate volatility impacts on above transportation-related outputs. The concept of vehicular jerk (derivative of acceleration) was explored [3, 4]. The findings showed the trend of vehicular jerk variation was identical for both bicycles and MVs regardless of roundabout design with a higher amplitude of variation for MVs. It was also found that the impact of MVs and bicycles speeds on driving volatility, as well as roundabout design was more important than bicycle volumes at roundabouts. Finally, the results of emissions dictated good relationships $\left(R^{2}>0.70\right)$ between acceleration and VSP modes distributions. The potential applications of this paper can be used for proving real-time information for drivers, or warning surrounding cyclists using emerging connected vehicle technologies. This paper also supplied relevant information for transportation experts to better understanding in how MV-bicycle interactions can affect traffic performance, safety, and emissions at circular intersections.
\end{abstract}

Keywords - Driving volatility; Cyclists; Roundabout; Safety; Emissions.

\section{ACKNOWLEDGMENT}

The authors acknowledge to the following projects: Centre for Mechanical Technology and Automation Strategic Project UID/EMS/00481/2019-FCT and CENTRO-01-0145FEDER-022083; @CRUiSE (PTDC/EMS-TRA/0383/2014), funded within Project 9471 - Reforçar a Investigação, o Desenvolvimento Tecnológico e a Inovação (Project 9471 RIDTI) and supported by European Community Fund FEDER; MobiWise (P2020 SAICTPAC/0011/2015), co-funded by COMPETE2020, Portugal2020 - Operational Program for Competitiveness and Internationalization (POCI), European
Union's ERDF (European Regional Development Fund), CISMOB (PGI01611, funded by Interreg Europe Programme) and FCT - Portuguese Science and Technology Foundation. The bicycle used in the data measurements was given by Orbita Bikes. The authors also acknowledge to Toyota Caetano Portugal, which allowed the use of vehicles and Pavlos Tafidis for his cooperation in data collection. This work was finally supported by the project POCI-01-0145-FEDER-029463, funded by FEDER, through COMPLETE2020-Portuguese Operacional Competitividade e Internacionalização (POCI), and by national funds (OE), through FCT/MCTES.

TOPIC

2) b.: Technologies for the Wellbeing - Innovative technologies for Smart Cities.

\section{REFERENCES}

[1] S.U. Jensen, "Safe roundabouts for cyclists," Accident Analysis \& Prevention, vol. 105, 2017, pp. 30-37.

[2] E. Ferguson, J. Bonneson, L. Rodegerdts, N. Foster, B. Persaud, C. Lyon, and D. Rhoades, "Development of Roundabout Crash Prediction Models and Methods," 2018 (No. Project 17-70).

[3] J. Liu, A.J. Khattak, and X. Wang, "A comparative study of driving performance in metropolitan regions using large-scale vehicle trajectory data: Implications for sustainable cities," International Journal of Sustainable Transportation, vol. 11(3), 2017, pp. 170-185.

[4] X. Wang, A.J. Khattak, J. Liu, G. Masghati-Amoli, and S. Son, "What is the level of volatility in instantaneous driving decisions?," Transportation Research Part C: Emerging Technologies, vol. 58, 2015, pp. 413-427. 